\title{
Correlations between microstructure and $Q$-factor of tunable thin film bulk acoustic wave resonators
}

\author{
A. Vorobiev, ${ }^{1, \text { a) }}$ S. Gevorgian, ${ }^{1}$ M. Löffler, ${ }^{2}$ and E. Olsson ${ }^{2}$ \\ ${ }^{1}$ Department of Microtechnology and Nanoscience, Chalmers University of Technology, \\ Gothenburg SE-41296, Sweden \\ ${ }^{2}$ Department of Applied Physics, Chalmers University of Technology, Gothenburg SE-41296, Sweden
}

(Received 9 February 2011; accepted 18 July 2011; published online 6 September 2011)

Correlations between microstructure and $Q$-factor of tunable solidly mounted $\mathrm{Ba}_{0.25} \mathrm{Sr}_{0.75} \mathrm{TiO}_{3}$ (BSTO) thin film bulk acoustic wave resonators are studied using analysis of test structures prepared at different growth temperatures of the BSTO films varying in the range $450-650{ }^{\circ} \mathrm{C}$. The observed changes in the $Q$-factor with growth temperature are correlated with related changes in microstructure, including the grain size, texture misalignment, interfacial amorphous layer, surface roughness, and deterioration of the Bragg reflector layers. The correlations are established through analysis of corresponding extrinsic acoustic loss mechanisms, including Rayleigh scattering at localized defects, acoustic attenuation by amorphous layer, generation of the shear waves leaking into the substrate, waves scattering by surface roughness, and resonance broadening by local thickness variations. It is shown that the waves scattering by surface roughness at the BSTO film interfaces is the main loss mechanism limiting the $Q$-factor of the BSTO thin film bulk acoustic wave resonators. (C) 2011 American Institute of Physics. [doi:10.1063/1.3626939]

\section{INTRODUCTION}

The electrically tunable thin film bulk acoustic wave (BAW) resonators, utilizing electric field induced piezoelectric effect in paraelectric phase ferroelectrics, enable development of novel reconfigurable/adaptable circuit architectures (e.g., agile front ends of microwave transceivers). However, the practical applications are hindered by their relatively low acoustic $Q$-factor. The product $Q \times f$, where $f$ is frequency, of the best reported tunable $\mathrm{Ba}_{\mathrm{x}} \mathrm{Sr}_{1-\mathrm{x}} \mathrm{TiO}_{3}$ (BSTO) BAW resonators, both membrane type and solidly mounted resonators (SMR), are in the range $400-600 \mathrm{GHz} .{ }^{1-4}$ Simulations show that product $Q \times f$ of a BAW resonator used in a ladder filter should be more than $2000 \mathrm{GHz} .^{5}$ For comparison, the nontunable BAW resonators utilizing AlN films reveal average $Q f \approx 3000 \mathrm{GHz}^{5}$ At the same time, extrapolation of $\mathrm{SrTiO}_{3}$ single crystal measurements gives $Q f \approx 6000 \mathrm{GHz}{ }^{6,7}$ This indicates that acoustic quality of the tunable BSTO BAW resonators is currently limited by extrinsic loss mechanisms, and there is room for its substantial improvement.

It is assumed that the extrinsic losses are caused by corresponding structural imperfections of the BSTO films. To the authors' knowledge, there is no publication devoted to a systematic study of correlations between microstructure and acoustic $Q$-factor of the BSTO BAW resonators. Some observations and assumptions are briefly discussed in a few papers. For example, transmission electron microscopy and $\mathrm{x}$ ray studies of the BSTO BAW resonators with rather low $Q f \approx 110 \mathrm{GHz}$ revealed a porous microstructure and random orientation of the BSTO grains. ${ }^{8}$ It is assumed that both lead to a variation of sound velocity and acoustic impedance throughout the film thickness, thus causing phase variations and scattering of acoustic waves, and as a consequence, a

${ }^{a)}$ Electronic mail: andrei.vorobiev@chalmers.se. reduction in the $Q$-factor. In Ref. 1 , we indicate that the acoustic attenuation in thin film materials is, in general, expected to be stronger than that in the bulk material counterparts due to scattering of the acoustic waves at film imperfections, such as voids, impurities, grain boundaries, etc. ${ }^{9}$ The BSTO films grown by pulsed laser deposition technique usually reveal relatively rough interfaces with peak-to-peak roughness of $5-10 \%$ of the film thickness. ${ }^{10}$ This results in loss due to leaking waves scattered by the surface roughness. Additionally, it is assumed that the columnar grains of the BSTO films with different heights and different crystallographic orientations (thus different acoustic velocities) would result in a distribution of closely spaced resonance frequencies and a lower overall $Q$-factor. ${ }^{10}$

In conventional, non-tunable AlN BAW-SMR, the extrinsic acoustic losses are associated mainly with the following leaking wave mechanisms: laterally leaking waves; waves scattered by surface roughness; and waves leaking through the Bragg reflector. ${ }^{5}$ Experiments have shown that lateral wave leakage is not the dominant loss mechanism in the BAW-SMR operating in the $Q$-regime up to 2500 . The main mechanism of scattering loss is the redirection of vertically moving acoustic energy toward lateral directions. This causes the waves to leave the active resonator region and dissipate either in the device substrate or in the region surrounding the device laterally. ${ }^{5}$ The scattering loss is usually negligible in AlN BAW-SMR, because even tens of nanometers interfacial roughness is relatively low in comparison with the AlN film thickness. For instance, the finite element simulations show that approximately $50 \mathrm{~nm}$ roughness of the Bragg reflector interfaces, which is only $1-2 \%$ of the piezoelectric film thickness, has no significant effect on the $Q$-factor of $1.9 \mathrm{GHz}$ AlN BAW-SMR. ${ }^{11}$ Generation of the shear waves and their leakage through the Bragg reflector into the substrate is a loss mechanism specific for the 
longitudinal acoustic mode BAW-SMR.,12 The origin of the shear waves is not well understood. ${ }^{12}$ Their generation is considered as a by-product of thickness expansional vibration. ${ }^{13}$ The loss due to shear waves leakage in AlN BAW-SMR has been reduced without improving the film microstructure, but modifying the Bragg reflector so that it works both for the longitudinal and the shear waves. After this modification, the $Q$-factor jumped from below 700 to above $1300 .{ }^{13}$ However, it is known that a piezoelectric thin film with mean/net tilt of the crystalline $c$-axis (rocking curve offset) generates shear waves. ${ }^{14,15}$ Furthermore, one can assume that each individual grain of a piezoelectric film with tilted c-axis produces a local shear wave. ${ }^{16}$ Thus, the loss associated with shear waves may be reduced by improving the film microstructure, namely, by reducing the c-axis rocking curve and the rocking curve offset.

In this paper, we present results of the correlated analysis of changes in $Q$-factor of the BAW-SMR and microstructure of the BSTO films, depending on the growth temperature of the BSTO films. The growth temperature is considered to be a key growth parameter, defining the BSTO film microstructure and, consequently, the acoustic performance. The analysis allows identification of the dominant extrinsic acoustic loss mechanisms. This, in turn, can help to further improve the $Q$-factor by optimization of the BSTO films' growth conditions and the BAW-SMR design. In the analysis, we consider waves scattering by localized defects, waves leaking through the Bragg reflector, waves scattering by surface roughness, and resonance broadening by local thickness variations. The losses associated with attenuation in amorphous interfacial layer and degradation of the Bragg reflector are also addressed.

\section{EXPERIMENTAL DETAILS}

The BSTO BAW-SMR test structures are fabricated on silicon substrates with resistivity $20 \mathrm{k} \Omega \cdot \mathrm{cm} .{ }^{16}$ The Bragg reflector, consisting of $\lambda / 4 \mathrm{SiO}_{2} / \mathrm{W}$ layers with thicknesses 280/240 $\mathrm{nm}$ and $100 \mathrm{~nm}$ Pt bottom plate, is deposited by magnetron sputtering. The BSTO films are grown by rf magnetron sputtering of the $\mathrm{Ba}_{0.25} \mathrm{Sr}_{0.75} \mathrm{TiO}_{3}$ target in on-axis configuration at $10 \mathrm{~cm}$ of the substrate-target distance. The template is heated by a radiative heater. The BSTO films for the BAW-SMR test structures are deposited at different growth temperatures in the range $450-650{ }^{\circ} \mathrm{C}$. The $\mathrm{Ar} / \mathrm{O}_{2}$ mixture with 1:1 flow ratio is used as a sputter gas at total pressure 2 mTorr. The $\mathrm{Al}$ top electrodes with $\mathrm{Ti}$ adhesion layer are deposited by e-beam evaporation. A lift-off process is used to pattern the top electrodes in the form of $60 \mu \mathrm{m}$ diameter central circular patches surrounded by $200 \mu \mathrm{m}$ diameter concentric outer electrodes. The relatively large area of the outer top electrodes provides effective dc and ac connection to the bottom plate. ${ }^{16}$ The capacitance of the BAWSMR test structures is measured at $1 \mathrm{MHz}$ using an HP 4285 A LCR-meter and a TTP6 Lake Shore probe station in the temperature range 50-300 K. In capacitance-voltage (permittivity-field) measurements, the dc bias is reversed periodically (two cycles) to record possible hysteresis effects. The complex input impedance $Z=R e Z+\mathbf{j} I m Z$ and admittance of the test structures are calculated using $S_{11}$ parameters measured by an Agilent N5230 A vector network analyzer and ground-signal-ground (GSG) microprobes in the frequency range 1-10 GHz. Modified Butterworth-Van Dyke (mBVD) circuit model is used for de-embedding of the electrical loss associated with series resistance $R_{S}$ composed by the ring section of the Pt bottom plate interconnect conductor and contact resistance between $\mathrm{Al}$ pads and probe tips. ${ }^{17}$ The series resistance in the mBVD model is found as the real part of impedance at the high frequency limit, which allows us to separate it from the equivalent resistance of BSTO capacitor associated with other non-ohmic contributions. ${ }^{18}$ The series resonance frequency $f_{s}$ is defined as frequency corresponding to the maximum of the real part of the admittance. The $Q$-factors at series $\left(Q_{s}\right)$ and parallel $\left(Q_{p}\right)$ resonances are evaluated as

$$
Q_{s, p}=\left.\frac{1}{2} f_{s, p} \frac{\partial \varphi}{\partial f}\right|_{f=f_{s, p}},
$$

where the phase angle is given as $\varphi=\operatorname{arctg}[\operatorname{ImZ} / \operatorname{ReZ}]$.

Microstructure of the BSTO films is analyzed using $\mathrm{x}$ ray diffraction (XRD), atomic force microscopy (AFM), and transmission electron microscopy (TEM) techniques. The XRD spectra are obtained using a Philips X'pert SW 3040 diffractometer equipped with a point $\mathrm{Cu} \mathrm{K}_{\alpha}$ radiation source, an MRD lens, a thin collimator, and a Ni filter. The BSTO film surface is characterized using a Digital Instruments Dimension 3000 scanning probe microscope (SPM) operating in tapping mode. The AFM images are analyzed using a scanning probe image processor (SPIP) 4.6.1.0 software tool. The TEM analysis was conducted on a Tecnai G2 T20 ST microscope at $200 \mathrm{kV}$ acceleration voltage, both in imaging and diffraction mode. Diffraction pattern analysis was conducted on averages of multiple selected area diffraction (SAD) patterns from the respective BSTO films. The samples for TEM analysis were prepared using focused ion beam (FIB) etching in a Fei Strata DB235 Dual Beam machine. Energy-dispersive $\mathrm{x}$ ray spectroscopy (EDX) is performed on the Pt layers in the TEM operating in scanning mode, using the electron beam as a local probe.

\section{RESULTS AND DISCUSSION}

\section{A. Evaluation of BSTO BAW-SMR test structure parameters}

As an example, Fig. 1 shows the measured reflection coefficients of a BAW-SMR test structure for 0,25 , and 50 $\mathrm{V}$ dc bias. The BSTO film is grown at $610^{\circ} \mathrm{C}$. The breakdown field of the BSTO films corresponds to approximately $60 \mathrm{~V}$. It can be seen that, without dc bias, the test structure reveals pure capacitive response. The resonance loop appears and develops with increasing dc bias due to the field induced piezoeffect.

For the purpose of establishing correlations between microstructure and $Q$-factor of the BSTO BAW-SMRs, it is constructive to distinguish between pure mechanical, dielectric, and electrical losses. In the mBVD equivalent circuit (Fig. 2(a)), these losses are represented by resistors $R_{m}, R_{0}$, and $R_{s}$, respectively. The series resistance $R_{S}$ in our BAW- 


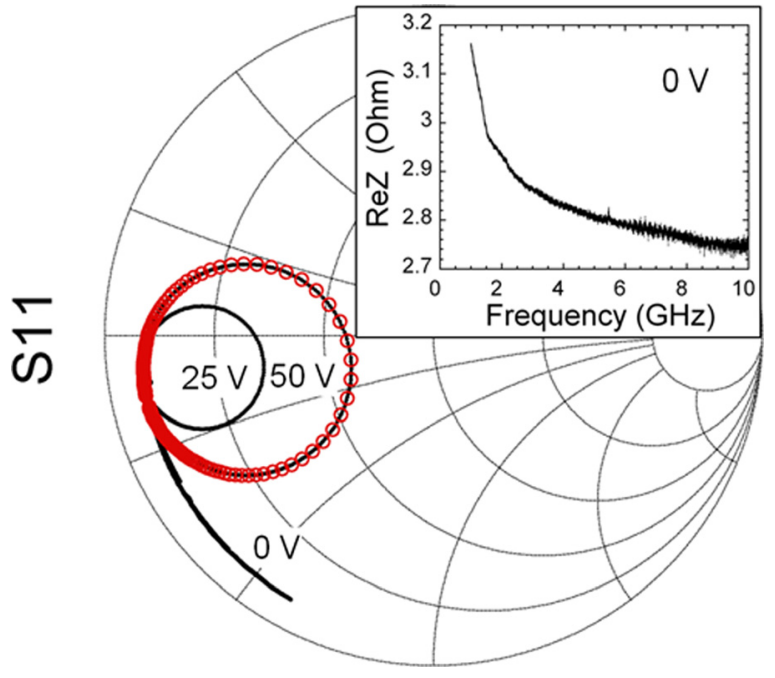

FIG. 1. (Color online) Measured reflection coefficients of a BAW-SMR test structure with the BSTO film grown at $610^{\circ} \mathrm{C}$ for 0,25 , and $50 \mathrm{~V}$ dc bias. Shown also is the loop (open circles) of the best fit to the mBVD model simulated using Agilent's ADS software. The inset shows real part of impedance vs frequency at $0 \mathrm{~V}$ dc bias.

SMR test structures is associated with the probe contacts and interconnects leading to a resonator. The definition in Eq. (1) allows expression of the $Q$-factors at series and parallel resonances in terms of $\mathrm{mBVD}$ circuit parameters as ${ }^{5}$

$$
\begin{aligned}
& Q_{s}=\frac{\omega_{s} L_{m}}{R_{s}+R_{m}}, \\
& Q_{p}=\frac{\omega_{p} L_{m}}{R_{0}+R_{m}} .
\end{aligned}
$$

It can be seen from Eq. (2) that the pure mechanical $Q$-factor can be evaluated using Eq. (1) at series resonance after deembedding the series resistance $R_{s}$ from the real part of impedance, i.e.,

$$
Q_{s-d e}=\left.\frac{1}{2} f_{s} \frac{\partial \varphi_{d e}}{\partial f}\right|_{f=f_{s}},
$$

where $\varphi_{d e}=\operatorname{arctg}\left[\operatorname{ImZ} /\left(\operatorname{ReZ}-R_{s}\right)\right]$. Below, we show that the $R_{S}$ can be estimated by analyzing the frequency dependence of the real part of impedance of the BSTO BAW-SMR test structures measured without dc bias. The corresponding equivalent circuit is shown in Fig. 2(b). In this case, without dc field, there is no motional branch, since there is no piezoeffect. We use representation of dielectric loss by parallel

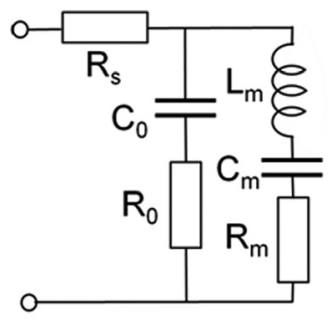

a

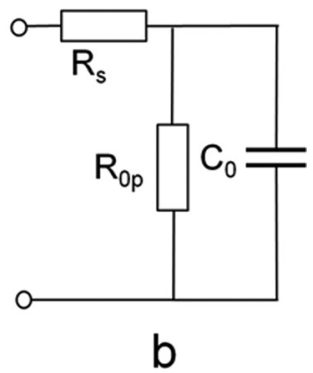

FIG. 2. The mBVD equivalent circuit of a piezoelectric resonator (a) and equivalent circuit of a BSTO BAW-SMR test structure without dc bias (b). resistance $R_{O p}$ because it gives a simpler form of the impedance. This representation, of course, does not change the $R_{s}$. The input impedance is

$$
Z_{0}=R_{s}+\frac{\frac{1}{R_{0 p}}}{\frac{1}{R_{0 p}^{2}}+\omega^{2} C_{0}^{2}}-j \frac{\omega C_{0}}{\frac{1}{R_{0 p}^{2}}+\omega^{2} C_{0}^{2}} .
$$

The loss tangent of a BSTO capacitor can be expressed as $\tan \delta=1 / \omega C_{0} R_{O p}$. On the other hand, it is known that both intrinsic and extrinsic microwave losses in a paraelectric phase BSTO are linearly proportional to the frequency and, hence, can be given as $\tan \delta=A \omega$, where $A$ is a function of a bias field. ${ }^{19}$ This leads to a rather simple form of the real part of impedance

$$
\operatorname{Re} Z_{0}=R_{s}+\frac{1}{A \omega^{2} C_{0}^{2}+\frac{1}{A}}
$$

It can be seen from Eq. (6) that, at high enough frequencies, the real part of impedance tends to the $R_{s}$. Our estimates indicate that the $R_{S}$ is frequency independent in the frequency range of interest, since the skin depth is much larger than the thickness of the interconnect plates. As an example, the inset in Fig. 1 shows the real part of impedance of the BAW-SMR test structure with the BSTO film grown at $610^{\circ} \mathrm{C}$ versus frequency at $0 \mathrm{~V}$ dc bias. It can be seen that, at frequencies above $8 \mathrm{GHz}$, the real part of impedance is rather frequency independent and, hence, $\operatorname{ReZ}=R_{s}$ which is $\sim 2.74 \Omega$.

Thus, the simple method given above allows establishing $R_{s}$. We have verified accuracy of the method by comparing $R_{s}$ with the value calculated correctly from the mBVD model (Fig. 2(a)). For calculations, we use standard definition of the effective coupling coefficient ${ }^{5}$

$$
k_{e f f}^{2}=\frac{\pi}{2} \frac{\omega_{s}}{\omega_{p}} \cot \left(\frac{\pi}{2} \frac{\omega_{s}}{\omega_{p}}\right) \approx \frac{\pi^{2}}{8} \frac{\omega_{p}^{2}-\omega_{s}^{2}}{\omega_{p}^{2}},
$$

which can be connected to the mBVD model parameters as

$$
k_{e f f}^{2}=\frac{\pi^{2}}{8} \frac{C_{m}}{C_{0}+C_{m}} .
$$

Then, the motional capacitance and inductance can be found using the following associations:

$$
\begin{gathered}
C_{m}=\frac{k_{\text {eff }}^{2}}{\frac{\pi^{2}}{8}-k_{e f f}^{2}} C_{0}, \\
L_{m}=\frac{1}{\omega_{s}^{2} C_{m}} .
\end{gathered}
$$

Further, the dc bias dependent dielectric capacitance and resistance can be expressed through the loss tangent measured at a dc bias, but at frequency $\omega$ well below resonances as

$$
\tan \delta_{V}=\omega C_{0}\left(R_{0}+R_{S}\right) .
$$

Solving a system of linear equations, Eqs. (2), (3), and (11) lead to 


$$
R_{s}=\frac{1}{2}\left[L_{m}\left(\frac{\omega_{s}}{Q_{s}}-\frac{\omega_{p}}{Q_{p}}\right)+\frac{\tan \delta_{V}}{\omega C_{0}}\right]
$$

and allows determining all parameters in the mBVD model. As an example, the parameters of the BAW-SMR test structure with the BSTO film grown at $610{ }^{\circ} \mathrm{C}$ measured at $50 \mathrm{~V}$ $\mathrm{dc}$ bias are summarized in Table I. The capacitance $C_{O}$ and loss tangent $\tan \delta_{\mathrm{V}}$ are measured at frequency $1 \mathrm{GHz}$. It can be seen that the $R_{s}$ value $2.5 \Omega$ is in good agreement with that found at the high frequency limit of the real part of impedance $2.74 \Omega$ (see inset in Fig. 1). Additionally, the measured one-port reflection coefficient data were fit to the mBVD model using Agilent's ADS software. The best fit loop is shown in Fig. 1 and resulted in the $R_{s}$ value $2.8 \Omega$, which also confirms our method of the $R_{s}$ evaluation.

Having all $\mathrm{mBVD}$ circuit parameters, one can define the purely mechanical $Q$-factor, also using Eq. (3), as

$$
Q_{m}=\frac{\omega_{p} L_{m}}{R_{m}} .
$$

Figure 3 shows the $Q$-factors of the BAW-SMR test structure with the BSTO film grown at $610^{\circ} \mathrm{C}$ versus dc bias voltage at series $\left(Q_{s}\right)$ and parallel $\left(Q_{p}\right)$ resonances calculated using Eq. (1). Shown also are the $Q$-factors at series resonance calculated using Eq. (4) after de-embedding the series resistance found at a high frequency limit of the real part of impedance (see inset in Fig. 1) $\left(Q_{s-d e}\right)$ and the mechanical $Q$-factor $\left(Q_{m}\right)$, calculated using Eqs. (13) and (12). The $Q$-factors approach zero at low fields, due to the nature of the field induced piezoeffect. It can be seen that, above $20 \mathrm{~V}$, the difference between $Q_{s-d e}$ and $Q_{m}$ is less than $10 \%$, which validates our simple method of the $R_{s}$ de-embedding. The $Q_{p}$ values are approximately 2 times lower than those of the $Q_{m}$, due to contribution of the dielectric loss, which is comparable with the pure acoustic loss (see $R_{O}$ and $R_{m}$ in the Table I). Our calculations using Eq. (11) give de-embedded loss tangent at $10 \mathrm{GHz} \tan \delta_{0} \approx 0.007$, which indicates rather high quality of the BSTO films, since it is only a few times higher than that of the $\mathrm{Ba}_{0.27} \mathrm{Sr}_{0.73} \mathrm{TiO}_{3}$ single crystal counterpart $\tan \delta_{\mathrm{sc}} \approx 0.002 .^{20}$ However, obviously, there is a room for further improvement of the BSTO film quality and, hence, decrease in the dielectric loss and corresponding increase in $Q_{p}$. It can be seen also from Fig. 3 that the $Q_{s}$ values are significantly lower than those of the $Q_{s-d e}$, which is caused by contribution of the electrical loss. From the device application point of view, it is important to analyze the origin of the electric loss. As it was mentioned, the series resistance $R_{S}$ in our BAW-SMR test structures is associated with the probe contacts and interconnects leading to a resonator. The interconnect part of the $R_{S}$ is composed mainly of a ring section of the Pt bottom plate and is given as ${ }^{21}$

TABLE I. The mBVD parameters of a BSTO BAW-SMR test structure.

\begin{tabular}{lccccc}
\hline \hline$R_{s}$ & $C_{0}$ & $R_{0}$ & $L_{m}$ & $C_{m}$ & $R_{m}$ \\
\hline $2.5 \mathrm{Ohm}$ & $5.4 \mathrm{pF}$ & $0.49 \mathrm{Ohm}$ & $4.5 \mathrm{nH}$ & $0.2 \mathrm{pF}$ & $0.6 \mathrm{Ohm}$ \\
\hline \hline
\end{tabular}

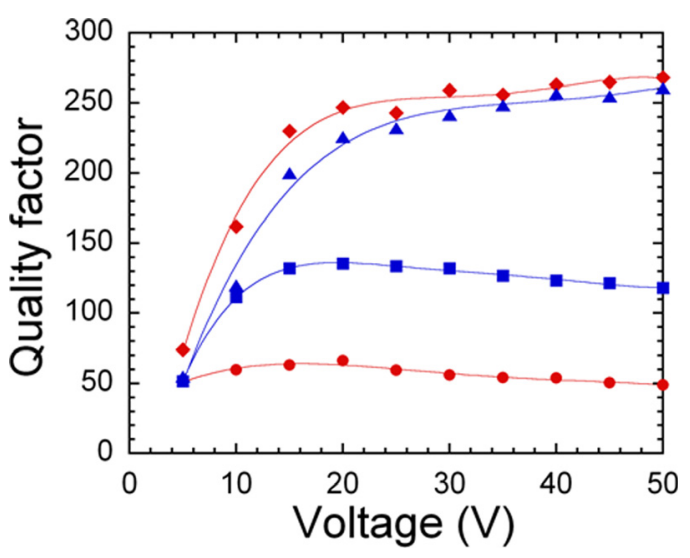

FIG. 3. (Color online) The quality factors at series (circles) and parallel (squares) resonances of a BAW-SMR test structure with the BSTO film grown at $610^{\circ} \mathrm{C}$ vs dc bias voltage. Shown also are the mechanical quality factors (triangles) and de-embedded quality factor at series resonance (diamonds).

$$
R_{\text {ring }}=\frac{R_{P t}}{2 \pi} \ln \left(\frac{b}{a}\right),
$$

where $R_{p t}$ is the sheet resistance of the Pt bottom plate and $a=30 \mu \mathrm{m}$ and $b=100 \mu \mathrm{m}$ are the radii of the central and outer electrodes, respectively. We have measured the $R_{p t}$ of a Pt film annealed at $610^{\circ} \mathrm{C}$ and the BSTO film growth conditions using the 4-point probe technique. The measured $\mathrm{Pt}$ sheet resistance is $3.6 \Omega$, which is almost 4 times larger than that of the bulk counterpart $1.06 \Omega$, most likely due to partial Pt oxidation during the BSTO film growth and formation of heterogeneous enclosures of $\mathrm{TiO}_{2-x}$ in the Pt layer, as follows from our XRD and EDX analyses. However, the corresponding $R_{\text {ring }}$ value calculated using Eq. (14) is only $0.7 \Omega$, which indicates that the major part of the $R_{S}(\sim 2.0 \Omega)$ is associated with the contact resistance between the probe tips and the $\mathrm{Al}$ electrodes of the BAW-SMR test structures.

Figure 4 shows the series and parallel resonance frequencies and their tunabilities measured at the BAW-SMR test structure with the BSTO film grown at $610^{\circ} \mathrm{C}$ versus dc bias voltage. Shown also is the effective coupling coefficient calculated using Eq. (7). It can be seen that the tunability of

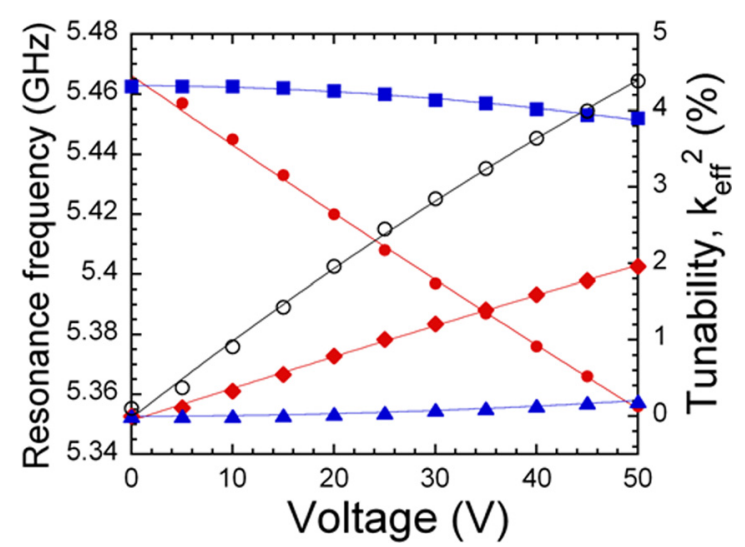

FIG. 4. (Color online) The series (circles) and parallel (squares) resonance frequencies and their tunabilities (diamonds and triangles, respectively) of a BAW-SMR test structure with the BSTO film grown at $610^{\circ} \mathrm{C}$ vs dc bias voltage. Shown also is the effective coupling coefficient (open circles). 
the series resonance frequency is $2 \%$ at $50 \mathrm{~V}$ dc bias. The effective coupling coefficient is $4.4 \%$.

\section{B. Microstructure}

Figure 5 shows the XRD patterns of the Bragg reflector stack (1), the Bragg reflector with Pt bottom plate (2), and the complete BAW-SMR test structure, including the BSTO film grown at $585^{\circ} \mathrm{C}$ (3). The Bragg reflector stack and Bragg reflector with Pt bottom plate are annealed at conditions of growth of the BSTO film. Shown also are the standard XRD data of $\mathrm{Ba}_{0.256} \mathrm{Sr}_{0.744} \mathrm{TiO}_{3}$ powder (indexed according to the International Centre for Diffraction Data (ICDD) entry 01-089-8211). It can be seen that there are no reflections from $\mathrm{BSTO}(\mathrm{h} 00)$ and (hk0) families of planes. The reflection from the BSTO(111) texture, confirmed by our SAD analysis, is masked by the strong $\mathrm{Pt}(111)$ and $\mathrm{W}(110)$ reflections (Fig. 5(b)). The typical halo reflection in the range $2 \theta=22-27^{\circ}$ (Fig. 5(a)) is attributed to the amorphous $\mathrm{SiO}_{2}$ matrix. The peaks marked as $\mathrm{c}(001), \mathrm{t}(101)$ and $\mathrm{q}(011)$ are identified as reflections of the strained cristobalite (indexed according to ICDD entry 76-0937), tridymite (indexed according to ICDD entry 89-3141), and quartz (indexed according to ICDD entry 88-2487), respectively.
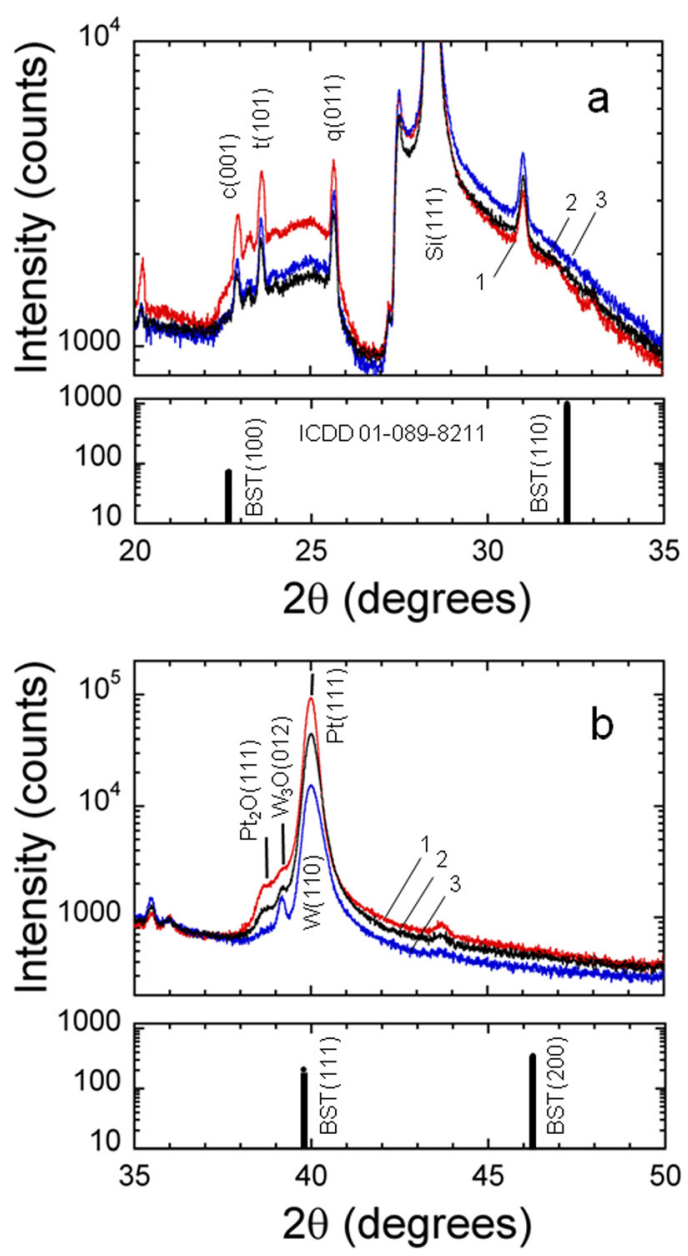

FIG. 5. (Color online) XRD patterns of a Bragg reflector stack (1), the Bragg reflector with Pt bottom electrode (2), and a complete BAW-SMR test structure, including the BSTO film grown at $585^{\circ} \mathrm{C}$ (3). Shown also are the standard XRD data of $\mathrm{Ba}_{0.25} \mathrm{Sr}_{0.75} \mathrm{TiO}_{3}$ powder.
This indicates that the $\mathrm{SiO}_{2}$ Bragg reflector layers are, partially, in crystalline state. The XRD spectra (Fig. 5(b)) reveal reflections from a low oxidized tungsten phase $\mathrm{W}_{3} \mathrm{O}(012)$ (indexed according to ICDD entry 41-1230), indicating that the W Bragg reflector layers are subjected to oxidation during the high temperature growth of the BSTO films. This is in agreement with severe oxidation of $\mathrm{W}$ observed after annealing of $\mathrm{W} / \mathrm{SiO}_{2}$ Bragg reflectors at $600^{\circ} \mathrm{C}^{22} \mathrm{The} \mathrm{SiO}_{2}$ recrystallization and $\mathrm{W}$ oxidation are presumably enhanced at higher growth temperatures of the BSTO films. According to the TEM cross-section, the $\mathrm{W}$ layers in the sample with BSTO film grown at $625^{\circ} \mathrm{C}$ seem to be approximately $10 \%$ thicker, which can be attributed to the $\mathrm{W}$ oxidation. ${ }^{22}$ Both recrystallization of the $\mathrm{SiO}_{2}$ and oxidation of the W layers potentially may result in deterioration of the reflection performance of the Bragg reflector and, hence, increase the losses associated with waves leaking into the substrate. The XRD spectra of samples containing Pt bottom plate reveal reflections from a low oxidized platinum $\mathrm{Pt}_{2} \mathrm{O}(111)$ phase (indexed according to ICDD entry 04-001-2426) (Fig. 5(b)). This means that the BSTO film growth conditions result in partial oxidation of the $\mathrm{Pt}$ bottom interconnect plate. It is assumed that $\mathrm{Pt}_{2} \mathrm{O}$ is a semiconductor with resistivity higher than that of the $\mathrm{Pt}$, as found for the higher oxidized platinum $\mathrm{PtO}_{\mathrm{x}}$ phases. ${ }^{23}$ Measurements of the Pt bottom plate interconnect sheet resistance using the 4-point probe technique give $R_{p t}=3.6 \Omega$, which is almost 4 times larger than that of the bulk counterpart $1.06 \Omega$. The increase in the series resistance due to platinum oxidation results in rise of the electrical loss and, hence, lower values of the device $Q$-factor. ${ }^{16}$

Figure 6 shows the AFM images of the BSTO films grown at $460^{\circ} \mathrm{C}, 610^{\circ} \mathrm{C}$, and $625^{\circ} \mathrm{C}$. Figure 7 shows the grain size and root mean square of the surface roughness of the BSTO films versus growth temperature obtained by SPIP analysis of the AFM images. It can be seen that the lateral grain size is in the range $20-40 \mathrm{~nm}$ and weakly depends on the growth temperature in the whole temperature range. The surface roughness of the BSTO films grown below $580^{\circ} \mathrm{C}$, defined mainly by the tips of the columnar grains, is in the range 2-3 nm and rather independent on the growth temperature. At higher growth temperatures, some craterlike depressions with lateral sizes $300-500 \mathrm{~nm}$ start to form and dominate the surface morphology. Consequently, the surface roughness increases sharply with the growth temperature up to $6 \mathrm{~nm}$ at $625^{\circ} \mathrm{C}$. It follows from the TEM analysis that formation and development of the craterlike depressions in the BSTO films may be associated with a deformation of the Pt bottom electrode. It will be shown in Subsection III C that the sharp increase in the surface roughness at higher growth temperatures correlates with an increase in a parameter of defect density in the BSTO material. The defects in the BSTO films are responsible for the acoustic loss due to Rayleigh scattering. ${ }^{24}$ The surface roughness itself results in acoustic loss, due to a generation of surface scattered leaking waves and loss due to resonance broadening caused by local variations of the film thickness. All these three types of acoustic losses, enhanced by increasing surface roughness, may be responsible for the observed drop in the acoustic $Q$ factor of the films grown above $580^{\circ} \mathrm{C}$. However, as it will 

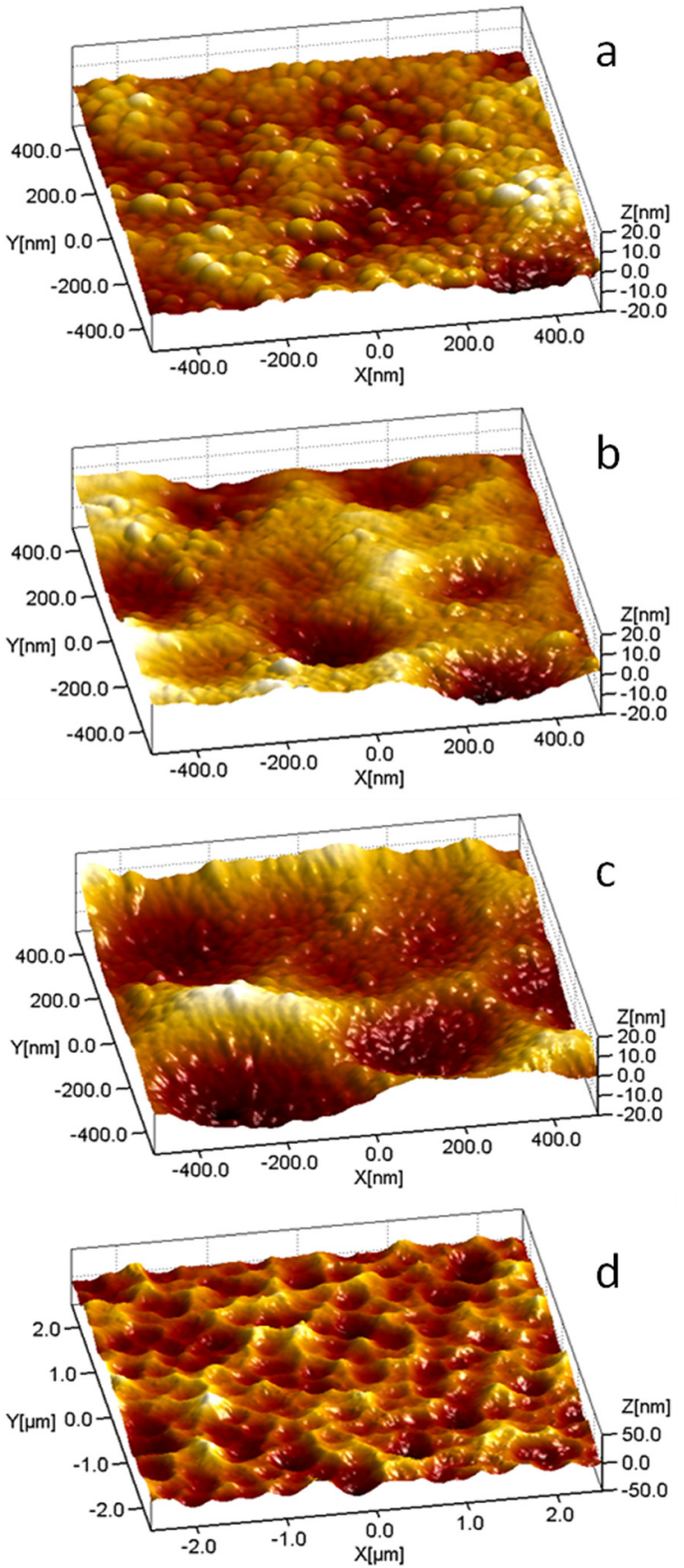

FIG. 6. (Color online) AFM images of the BSTO films grown at $460^{\circ} \mathrm{C}$ (a), $610^{\circ} \mathrm{C}(\mathrm{b})$, and $625^{\circ} \mathrm{C}(\mathrm{c})$ and (d).

be shown by simulations, the scattering by surface roughness is the dominant loss mechanism for films grown above $580^{\circ} \mathrm{C}$

The TEM cross section analysis is an extremely useful supplement to AFM and XRD data, since it allows analyzing the microstructure of the BSTO film, the bottom interface of the BSTO film, the morphology of the bottom Pt electrode, and provides knowledge about changes in the Bragg reflector stack. The results obtained by TEM studies are presented in Fig. 8 and Fig. 9 as well as in Table II.

Texturing is a phenomenon closely linked to surface energies, thermal strains, grain boundary energies, and mobilities of the atoms during growth. The BSTO films show (111) texturing parallel to the growth direction (Fig. 8(c)).

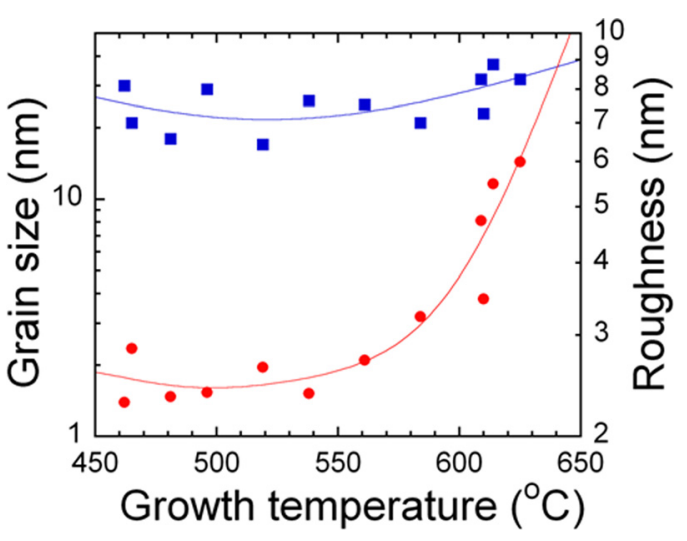

FIG. 7. (Color online) In-plane grain size (squares) and root mean square of the surface roughness (circles) of the BSTO films vs growth temperature. The lines are polynomial curve fits.

The extracted rocking curve is obtained by assuming a 2DGaussian distribution of (111) around the growth direction. The electron diffraction patterns originate from the grains, which are aligned around a 1D cut through this distribution. The intensity profiles of the (111) arc pattern were fitted by
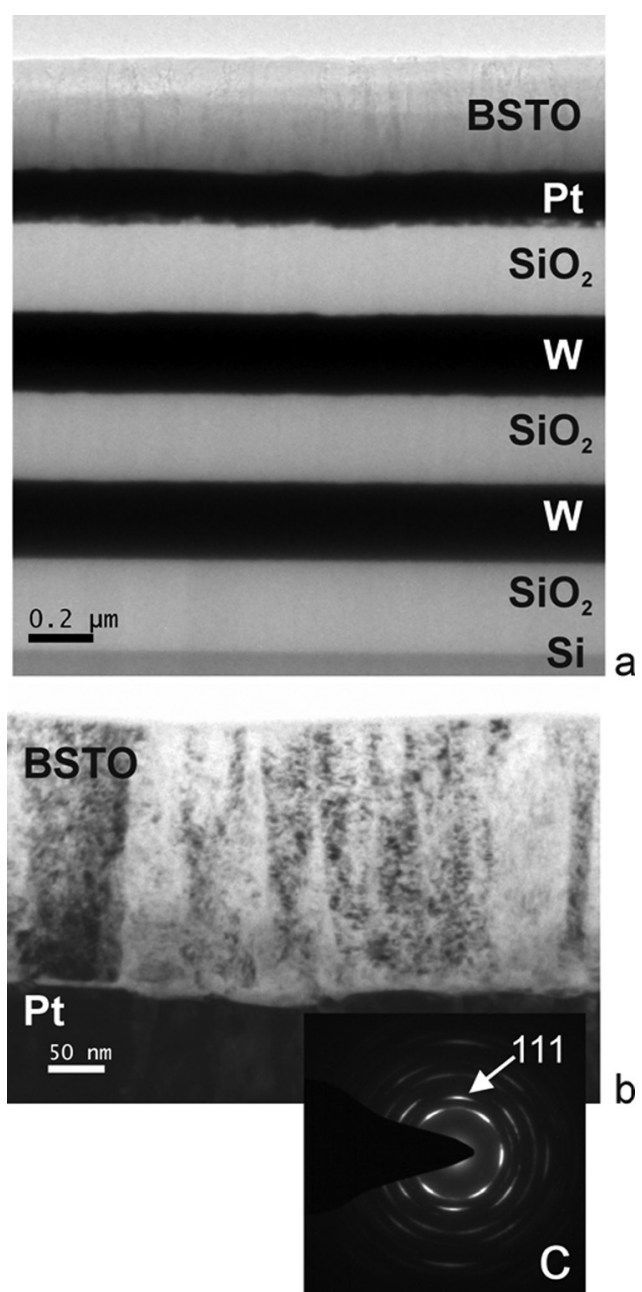

FIG. 8. TEM images of a BAW-SMR test structure with the BSTO grown at $585^{\circ} \mathrm{C}$ : cross-section image of the whole structure, including the $\mathrm{W} / \mathrm{SiO}_{2}$ Bragg reflector, the Pt bottom electrode, and the BSTO film (a); cross-section image of the Pt layer and the BSTO film with different grains visible by changes in the contrast resulting from different orientations (b); SAD-pattern of the BSTO film averaged over several positions on the film cross-section (c). 


$$
y=y_{0}+\frac{A}{w \sqrt{\pi / 2}} \exp \left[-2 \frac{\left(x-x_{0}\right)^{2}}{w^{2}}\right],
$$

where $y_{0}$ is an offset, $A$ is an amplitude, $x_{0}$ is the center of the peak, and $w$ is its width.

The misalignment angle, defined as the width of the distribution, is minimal for the sample grown at $585^{\circ} \mathrm{C}$ and larger for lower and higher temperatures (Table II). This dependence will be explained by taking both the crystallite growth as well as the templating by the bottom electrode into account.

Lateral grain sizes have been determined by measuring the average length between contrast changes while using a small objective aperture. Contrast changes indicate different grain orientations. A similar technique was used to assess the "columness" of the BSTO film, i.e., the ratio between columns, which change contrast in growth direction and columns without contrast change. Both numbers are, of course, only to be taken as an approximate means and do not give exact numbers, since the results are skewed by, e.g., overlapping grains in electron beam direction as well similarly oriented grains, which do not show contrast differences between them. However, several trends may be observed from this analysis. We can see that the lateral grain size distribution increases with temperature, both in mean as well as in width. Furthermore, the "columness" is the same for the samples grown at $585^{\circ} \mathrm{C}$ and $625^{\circ} \mathrm{C}$, while it is much lower for the sample grown at $495^{\circ} \mathrm{C}$. This could indicate that, in the latter sample, nucleation processes compete with grain growth, leading to the nucleation of the new grains on top of the other grain. This process seems to be much less pronounced at higher growth temperatures.

The interface between the Pt layer and the BSTO films shows an amorphous layer at $495^{\circ} \mathrm{C}$, which is reduced in thickness at $585^{\circ} \mathrm{C}$ and not observable anymore at $625^{\circ} \mathrm{C}$ (Table II and Fig. 9). However, occasionally, small localized patches of an amorphous layer were observed at steep edges in the BSTO/Pt interface. This amorphous layer is assumed to be composed of oxides of the BSTO components.

While the sample grown at $495^{\circ} \mathrm{C}$ shows a rather smooth Pt-layer with occasional steps and small height changes, the sample grown at $625^{\circ} \mathrm{C}$ shows a recrystallization of the $\mathrm{Pt}$ layer with large hillocks and large height changes (see Fig. 9). This results in a rough bottom interface, which in turn can nucleate tilted grains. Since the BSTO film has no cracks or voids, one may assume that at least a part of the transformation in the bottom electrode takes place before any significant BSTO growth starts. In essence, during heatup, ridges are formed in the Pt film (Fig. 9). Once the BSTO deposition starts, the grains grow on this corrugated film, reproducing the morphology. Individual protrusions in the bottom electrode can be tens of nm high and therefore offer tilted faces for grain nucleation. While the surface roughness of the bottom electrode, i.e., at the lower side of the BSTO film, increases with temperature up to about $40 \mathrm{~nm}$, the root mean square (estimated by TEM cross section, Table II), the surface roughness of the top side reaches only values of about $6 \mathrm{~nm}$ at $625^{\circ} \mathrm{C}$ (Fig. 7). The increase in top surface

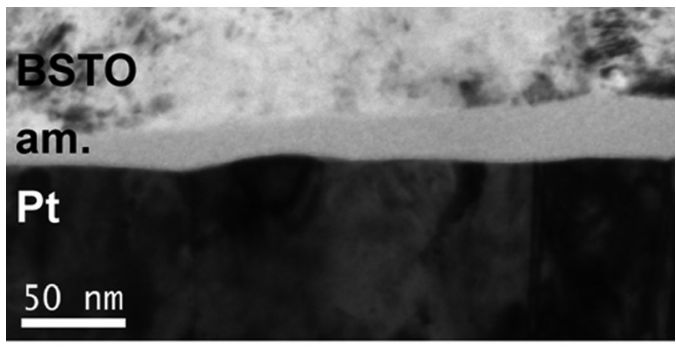

$495^{\circ} \mathrm{C}$
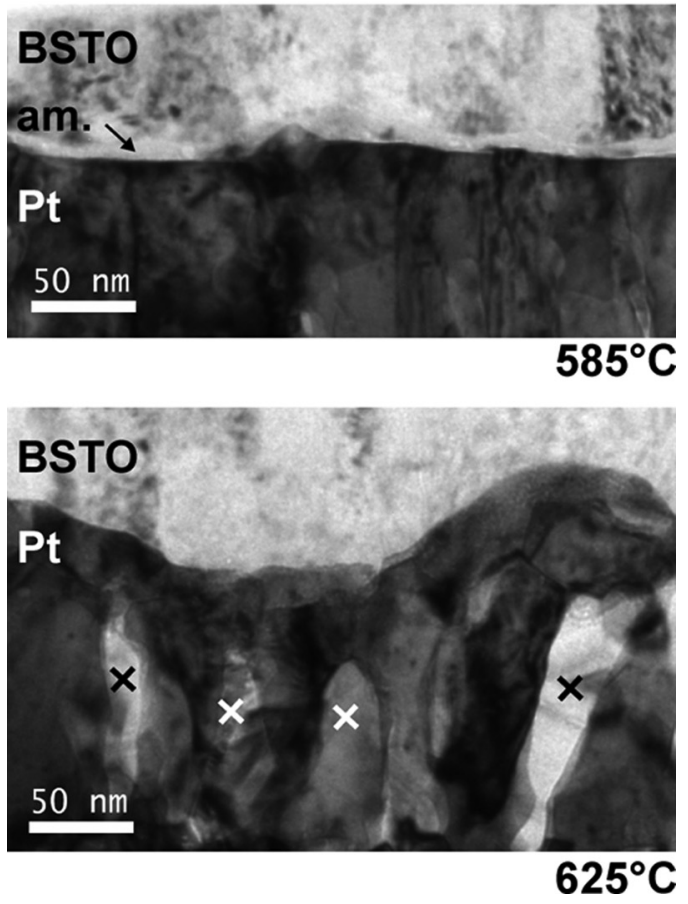

FIG. 9. TEM cross-section images of the BSTO/Pt interface for different BSTO film growth temperatures, showing changes in the interface morphology, amorphous layer (denoted as am.) thickness, and formation of $\mathrm{TiO}_{2-\mathrm{x}}$ enclosures (marked by crosses) in the Pt layer.

roughness, as measured by AFM, can therefore be considered as a result of the changes in the underlying bottom electrode morphology. In fact, some coincidence was found between a large hillock in the bottom electrode and a ridge on the top surface; however, the height of the ridges is lower on the surface of the BSTO.

It can be seen from Fig. 9(c) that the hillocks in the Pt layer contain heterogeneous enclosures, which we identified by EDX analysis as $\mathrm{TiO}_{2-\mathrm{x}}$ grains. We assume that, during high temperature growth of the BSTO film, the Ti from adhesion layer diffuses intensively along the Pt columnar grain boundaries, while oxygen is propagating in the opposite direction. Oxidation of $\mathrm{Ti}$ inside the Pt layer results in formation of $\mathrm{TiO}_{2-x}$ grains, volume expansion, and deformation of the Pt electrode. ${ }^{25}$

The above analysis allows us to conclude that the observed broadening of the rocking curve at low growth temperatures (Table II) is, most likely, a result of the low mobility of the constituting atoms. This is also supported by the higher amount of the new grains nucleated on top of other grains, which points to conditions where nucleation and continued grain growth compete and, therefore, can lead to a larger overall misalignment. The misalignment at 
TABLE II. Structural parameters of the BSTO films grown at different temperatures.

\begin{tabular}{lcccc}
\hline \hline & $\begin{array}{c}\text { Thickness of } \\
\text { amorphous } \\
\text { layer (nm) }\end{array}$ & $\begin{array}{c}\text { Texture } \\
\text { misalignment } \\
(\mathrm{deg})\end{array}$ & $\begin{array}{c}\text { BSTO lateral } \\
\text { grain size (nm) }\end{array}$ & $\begin{array}{c}\text { Bottom interface } \\
\text { roughness (nm) }\end{array}$ \\
\hline 495 & 14.4 & 16.5 & 43 & 5 \\
585 & 6 & 15.35 & 48 & 14 \\
625 & $\leq 1^{\mathrm{a}}$ & 17.85 & 53 & 39 \\
\hline \hline
\end{tabular}

${ }^{\mathrm{a}}$ The amorphous layer is not observable, partly due to large surface roughness.

higher temperatures is most likely a secondary effect and can be explained as a result of the inclined templation at the rough bottom electrode. Both phenomena lead to a broader distribution of (111) orientations. With the reduced misalignment, one can expect a reduction in the generation of acoustic shear waves and, correspondingly, an increase in the acoustic $Q$-factor for the films grown at higher temperatures. This will be discussed later.

The Bragg reflector stack shows smooth interfaces between $\mathrm{W}$ and $\mathrm{SiO}_{2}$ for all three samples. However, the thickness of W with respect to $\mathrm{SiO}_{2}$ is increased for the sample grown at $625^{\circ} \mathrm{C}$, indicating partial oxidation of $\mathrm{W}$. This confirms the results of the XRD analysis discussed above. These changes in acoustic properties may potentially lead to deterioration of the reflection coefficient of the Bragg reflector and result in increased loss associated with waves leaking into the substrate.

\section{Dielectric response}

Figure 10 shows the temperature dependence of the apparent (measured) permittivity of a BSTO film grown at $585^{\circ} \mathrm{C}$. The dependence differs from that of the single crystal counterpart, which reveals sharp maximum at $T_{m}=125 \mathrm{~K}$ and a room temperature permittivity of $460 .^{20,26}$ The apparent permittivity of the BSTO film is reduced and reveals a diffuse maximum shifted to a higher temperature $T_{m}=150 \mathrm{~K}$. This is a typical effect of the ferroelectric material inhomogeneities. The inhomogeneities result in formation of the residual polar regions and, consequently, broaden the phase transition and

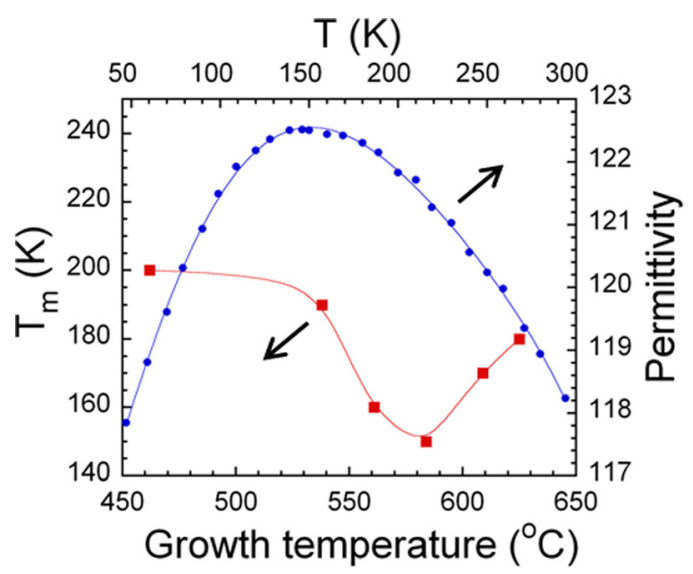

FIG. 10. (Color online) Apparent permittivity of a BSTO film grown at $585^{\circ} \mathrm{C}$ (circles) vs temperature of measurement (upper abscissa) and temperature of maximum of the permittivity $T_{m}$ (squares) vs growth temperature. The lines are curve fits. shift $T_{m}$ to the higher temperature. ${ }^{27}$ The $T_{m}$ value of a real, defected crystal without external field can be expressed $\mathrm{as}^{27}$

$$
T_{m}=T_{C} \sqrt{\left[\left(\frac{1}{2} \xi_{s}\right)^{2 / 3}+1\right]^{2}-\left(\frac{\theta_{F}}{4 T_{C}}\right)^{2}}
$$

where $T_{c}$ is the Curie temperature and $\theta_{F}$ is the effective Debye temperature. Parameter $\xi_{s}$ is the normalized statistical dispersion of the built-in field produced by the structural imperfections, such as grain boundaries, strain, and other defects. ${ }^{27,28}$ Thus, the parameter $\xi_{s}$ can be considered as a parameter of defect density in the BSTO material. For single crystals, $\xi_{s}=0.01-0.05$, and for ceramic samples, $\xi_{s}=0.1-1.5 .^{27}$ Fig. 10 depicts also temperatures of maximum permittivity $T_{m}$ of the films deposited at different growth temperatures. It can be seen from Fig. 10 that there is a clear dependence of $T_{m}$ and, hence, the parameter of defect density $\xi_{s}$ on the growth temperature. Figure 11 shows the parameter of defect density $\xi_{s}$ calculated using Eq. (16), assuming $\theta_{F}=175 \mathrm{~K}$ and $T_{c}=122 \mathrm{~K}^{20,27}$ As it can be seen, $\xi_{s}$ and, hence, density of defects in the BSTO films decreases with the growth temperature up to, approximately, $T_{g}=580^{\circ} \mathrm{C}$. It was shown that the oxygen vacancies are inherent for BSTO. ${ }^{29}$ These defects are located within the non-stoichiometric grain boundaries. ${ }^{30}$ The presence of the oxygen vacancies, in particular, may result in the hysteresis behavior of the field dependence of permittivity. ${ }^{31,32}$ The analysis of the dielectric response of our samples shows that the hysteresis phenomenon is well pronounced in the BSTO films grown at low temperatures and diminishes gradually with growth temperature in the whole temperature range. For example, Fig. 12 shows the apparent permittivity of our BSTO films grown at $460^{\circ} \mathrm{C}$ and $625^{\circ} \mathrm{C}$ versus electrical field. Diminishing of the hysteresis indicates that the density of the localized defects, which can be attributed to the oxygen vacancies, decreases gradually with growth temperature. This is in agreement with TEM analysis, revealing increased lateral sizes of the BSTO grains with growth temperature (Table II). In other words, the relative amount of the grain boundaries decreases with growth temperature, resulting in a decrease in the amount of oxygen

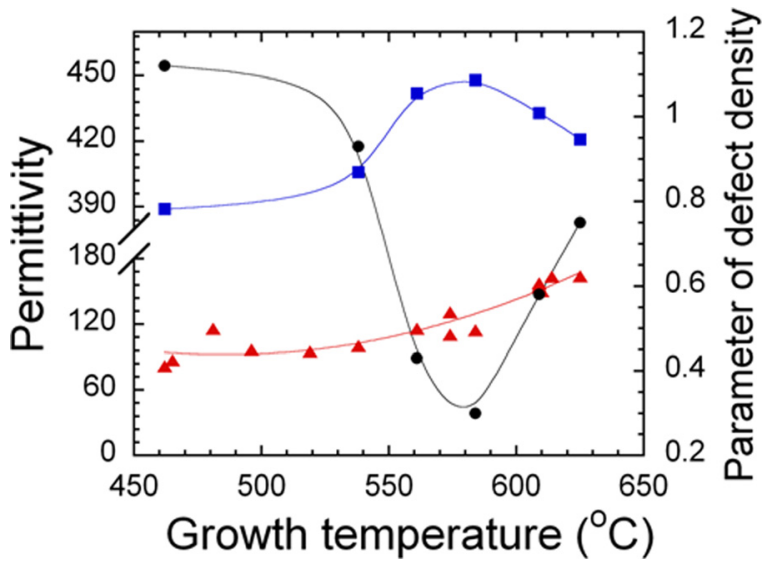

FIG. 11. (Color online) Apparent (triangles) and bulk (squares) permittivity and parameter of defect density $\xi_{s}$ (circles) of the BSTO films vs growth temperature. The lines are curve fits. 


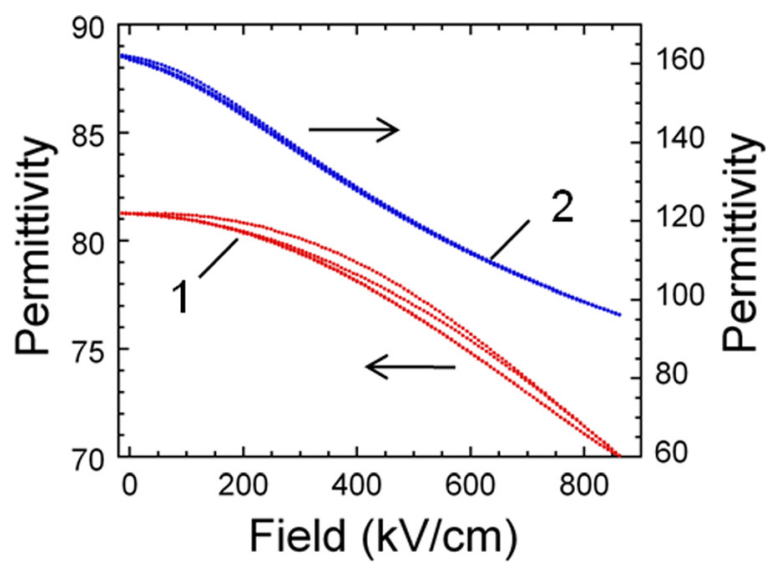

FIG. 12. (Color online) Electric field dependences of the apparent permittivity of the BSTO films grown at $460{ }^{\circ} \mathrm{C}$ (1) and $625^{\circ} \mathrm{C}(2)$.

vacancies accommodated there. The increase in the parameter of defect density with growth temperatures above $T_{g}=580^{\circ} \mathrm{C}$ can be correlated with the development of other types of inhomogeneities, for example, local structure damages caused by the increase in roughness at the bottom (Table II) and top interfaces (Fig. 6 and Fig. 7).

Having the parameter of defect density $\xi_{s}$, the permittivity of the BSTO without external field may be given as ${ }^{27}$

$$
\begin{gathered}
\varepsilon=\varepsilon_{00} / \Phi \\
\Phi=\left[\left(\xi_{s}^{2}+\eta^{3}\right)^{1 / 2}+\xi_{s}\right]^{2 / 3}+\left[\left(\xi_{s}^{2}+\eta^{3}\right)^{1 / 2}-\xi_{s}\right]^{2 / 3}-\eta \\
\eta=\sqrt{\left(\frac{\theta_{F}}{4 T_{C}}\right)^{2}+\left(\frac{T}{T_{C}}\right)^{2}}-1
\end{gathered}
$$

where $\varepsilon_{00}=C / T_{C}$ and $C$ is the Curie constant. Figure 11 shows the permittivity calculated using Eqs. (17)-(19), $T=295 \mathrm{~K}$, and $C=8 \times 10^{4} \mathrm{~K}^{20}$ It can be seen that the BSTO films with the lowest parameter of defect density, grown in the temperature range $T_{g}=550-600^{\circ} \mathrm{C}$, reveal permittivity approaching that of the single crystal counterpart, i.e., $\varepsilon=460$. This indicates that the BSTO films grown in this temperature range possess almost defectless volume. However, the apparent permittivity exhibits much lower values (Fig. 11). Typically, a thin film reveals lower permittivity than that of the bulk counterpart, with the same composition and defect population, due to several physical size effects, including the surface blocking of polarization, electrode and depletion effects, misfit strain gradient, and presence of a secondary phase at interfaces. ${ }^{19}$ In particular, our TEM analysis clearly indicates the presence of an amorphous layer at the bottom interface (Fig. 9). One can assume that the amorphous layer is formed mainly by oxides of the BSTO components $\mathrm{TiO}_{2}$ and $\mathrm{SrO}$ with permittivity $\varepsilon_{i a} \approx 13 .^{33,34}$ Then, the apparent permittivity can be modeled, assuming in-series connection of the ferroelectric film with the lower permittivity interfacial layer. Our calculations indicate that the amorphous layer contributes significantly to the total size effect. The observed increase in the apparent permittivity with growth temperature can be explained by corresponding decrease in the amorphous layer thickness (Table II).

\section{Quality factor}

Figure 13 shows the BSTO film growth temperature dependent $Q$-factors of the BAW-SMR test structures at $25 \mathrm{~V}$ dc bias calculated using Eq. (4) after de-embedding the series resistance found at high frequency limit of the real part of impedance. A rather sharp maximum, with $Q$-factors up to 400 at $T_{g} \approx 590{ }^{\circ} \mathrm{C}$, can be observed. It is assumed that, at lower and higher growth temperatures, the $Q$-factors are reduced, due to enhanced extrinsic acoustic losses associated with an increased amount of the BSTO film structural imperfections. The relevant structural imperfections and their development with BSTO film growth temperature are analyzed in Subsections III B and III C. In this section, we present a correlated analysis of the changes in $Q$-factor of the BAWSMR and the microstructure of the BSTO films, depending on the growth temperature. The analysis allows identification of the dominant extrinsic acoustic loss mechanisms. This, in turn, may help to further improve the $Q$-factor through optimization of the BSTO film growth conditions and the BAWSMR design. In the analysis, we will consider all extrinsic acoustic loss mechanisms, which, we assume, can contribute in the total loss balance of our BSTO BAW-SMR: waves scattering by localized defects; attenuation by amorphous interfacial layer; waves leaking through the Bragg reflector; waves scattering by surface roughness; and resonance broadening by local thickness variations.

It can be seen, from comparison of Fig. 13 and Fig. 11, that the increase and following decrease in the $Q$-factor with growth temperature below and above $T_{g} \approx 590{ }^{\circ} \mathrm{C}$ correlate well with corresponding decrease and increase in the $\xi_{s}$ parameter, which is a quantitative characteristic of density of defects in the BSTO material. As it was shown in Subsection III $\mathrm{C}$, the decrease in the parameter of defect density with growth temperature can be correlated with reduction in density of oxygen vacancies presumably located within the nonstoichiometric grain boundaries. The presence of localized defects in an elastic medium gives rise to a sound attenuation, known as Rayleigh scattering. ${ }^{24}$ Thus, the observed increase in the $Q$-factor with growth temperature can be correlated

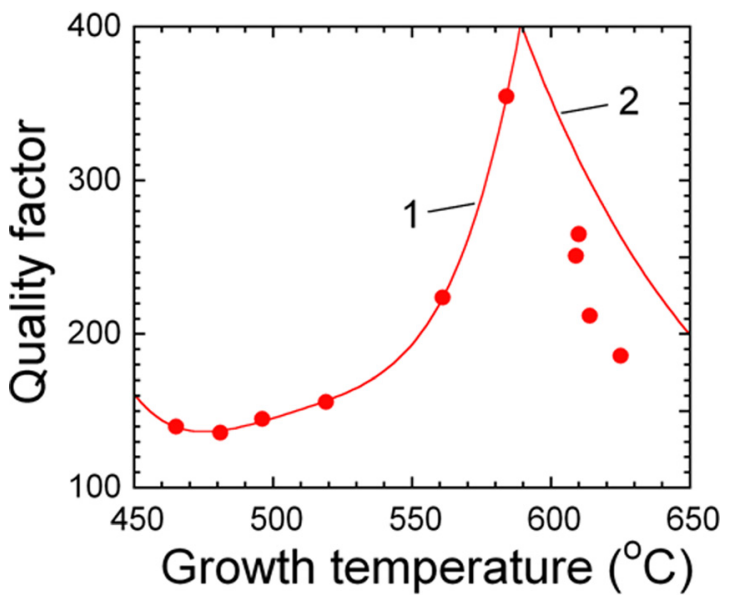

FIG. 13. (Color online) De-embedded quality factor (circles) vs growth temperature. The line 1 is a polynomial curve fit. The line 2 is a simulated quality factor, taking into account loss associated with scattering by surface roughness. 
with reduction in loss by Rayleigh scattering at oxygen vacancies. As it was mentioned, the subsequent increase in the parameter of defect density above $T_{g}=580^{\circ} \mathrm{C}$ can be associated with development of other types of inhomogeneities, for example, local structure damages caused by an increase in roughness at bottom (Table II) and top interfaces (Fig. 6 and Fig. 7), which is not necessarily resulting in a remarkable acoustic loss.

The increase in the $Q$-factor with growth temperature correlates also with decrease in the thickness of the amorphous layer at the bottom interface, as detected by TEM analysis (Fig. 9 and Table II). The analysis of the size effect indicates significant influence of the amorphous interfacial layer on the dielectric response. The strong increase in the sound attenuation in the amorphous state is demonstrated by various models for the vibrations in disordered systems. ${ }^{35,36}$ Thus, we assume that the observed increase in the $Q$-factor of the BAW-SMR with growth temperature of the BSTO films is associated also with reduction in the amorphous layer thickness and, thereby, reduction in the acoustic attenuation in this amorphous layer.

The generation of shear waves leaking through the Bragg reflector into the substrate is a loss mechanism specific for longitudinal mode SMRs. ${ }^{5,12}$ It is shown that a piezoelectric thin film with mean/net tilt of the crystalline $c$-axis (rocking curve offset) generates shear waves. ${ }^{14,15}$ Further, we assume that each individual grain of a piezoelectric film with tilted caxis produces a local shear wave as well. ${ }^{16}$ Typically, the rocking curve, which is a measure of the crystalline $c$-axis out-of-plane misorientation, decreases with growth temperature. ${ }^{37}$ In accordance with our SAD analysis, the BSTO film grown at $T_{g}=585^{\circ} \mathrm{C}$ reveals the lowest (111) texture misalignment (Table II). Thus, the observed increase and following decrease in the $Q$-factor with growth temperature below and above $T_{g} \approx 590^{\circ} \mathrm{C}$ (Fig. 13) can be correlated with corresponding decrease and increase in the generation of shear waves. However, our recent acoustoelectric modeling indicates that the generation of shear waves leaking through the Bragg reflector is likely not a dominant loss mechanism in our BSTO BAW-SMR. One can partly attribute the drop in the $Q$-factor above $T_{g} \approx 590{ }^{\circ} \mathrm{C}$ to a degradation of the Bragg reflector performance. In accordance with our XRD and TEM analysis, the $\mathrm{SiO}_{2}$ and $\mathrm{W}$ Bragg reflector layers are subjected, respectively, to recrystallization and partial oxidation during the high temperature growth of the BSTO films in oxygen ambient. Both effects potentially result in a deterioration of the reflection performance and, hence, an increase in loss associated with waves leaking through the Bragg reflector into the substrate. This is in agreement with oxidation of $\mathrm{W}$ and corresponding reduction in BAW-SMR $Q$-factor after annealing of $\mathrm{W} / \mathrm{SiO}_{2}$ Bragg reflectors at $600^{\circ} \mathrm{C} .^{22}$

The observed drop in the $Q$-factor with growth temperature above $T_{g} \approx 590{ }^{\circ} \mathrm{C}$ can be correlated with a rapid increase in the BSTO films roughness at bottom (Table II and Fig. 9) and top interfaces (Fig. 6 and Fig. 7), resulting in intensive scattering of the acoustic waves. Theory of diffused reflection/scattering of the acoustic waves from the surface of a rough mirror is proposed in Ref. 38. Using the results of this theory, a simple approximation for the loss tangent asso- ciated with the diffuse scattering of the acoustic energy from the rough top interface can be deduced:

$$
\tan \delta_{s c a t}=\left[1-\exp \left(-2 \pi k_{a c}^{2} \sigma^{2}\right)\right] \frac{d}{\sigma \sqrt{8 \pi^{3}}},
$$

where $d$ is the mean diameter of the surface features and $\sigma$ is the standard deviation of the roughness height. The acoustic wavenumber $k_{a c}$ is defined as

$$
k_{a c}=\frac{\omega}{v_{a c}},
$$

where $v_{a c}=8141 \mathrm{~m} / \mathrm{s}$ is the acoustic velocity. ${ }^{39}$ Notice that Eq. (20) takes into account a single act of scattering from one interface only. It is assumed also that the scattering losses due to the multiple reflections may be ignored. The part of the scattered energy coupled back is also ignored. In calculation of scattering losses in Eq. (20), the acoustic velocity is assumed to be constant and dc bias independent. We consider that the total $Q$-factor of the BSTO BAWSMR $Q_{\text {tot }}$ includes two terms: structural, $Q_{s t r}$, given by fitting curve 1 in Fig. 13 and scattered $Q_{\text {scat }}=1 /$ tan $\delta_{\text {scat }}$. The $Q_{s t r}$ increases with growth temperature, due to improvement in the BSTO film structural quality. In general, the energies $E_{\text {scat }}$ and $E_{\text {str }}$ associated with corresponding loss mechanisms are different and ${ }^{5}$

$$
\begin{gathered}
\frac{1}{Q_{\text {tot }}}=\frac{1}{E_{\text {tot }}}\left(\frac{E_{\text {str }}}{Q_{\text {str }}}+\frac{E_{\text {scat }}}{Q_{\text {scat }}}\right), \\
E_{\text {tot }}=E_{\text {str }}+E_{\text {scat }} .
\end{gathered}
$$

We assume, as a first approximation, that for films grown above $T_{g} \approx 590^{\circ} \mathrm{C}$, the ratio $E_{\text {scat }} / E_{\text {str }}$ equals to the relative area occupied by the craterlike depressions. The relative area and the mean diameter of the crater-like depressions are found using the SPIP analysis of the AFM images. The curve 2 in Fig. 13 represents the $Q_{\text {tot }}$ calculated using Eqs. (20)-(23). It can be seen that modeling of the loss due to a scattering by surface roughness describes well the observed drop in the $Q$-factor with growth temperature above $T_{g} \approx 590{ }^{\circ} \mathrm{C}$. The calculated $Q$-factor associated with scattering is overestimated, partly because the model does not take into account scattering from the bottom interface, which actually reveals an even larger roughness (Table II and Fig. 9). Besides, there are probably other loss mechanisms responsible for the decrease in the $Q$-factor with growth temperature above $T_{g} \approx 590^{\circ} \mathrm{C}$.

The resonance broadening by local thickness variations is another effect, which can be associated with surface roughness and presumably contributing to the drop in the $Q$-factor above $T_{g} \approx 590^{\circ} \mathrm{C}$. A BSTO film can be modeled as an assembly of nanosize columns, grains, or grain complexes, which are considered to be individual nanoresonators. ${ }^{10}$ The nanoresonators are assumed to have the same acoustic and dielectric properties but different heights and, thus, closely spaced but different resonance frequencies, i.e., the assembly has a resonance frequency distribution. This results in broadening of a BAW-SMR resonance curve, which, in accordance 
with our recent modeling, may result in a drastic reduction in the overall $Q$-factor of the BAW-SMR.

\section{SUMMARY}

We present results of the correlated analysis of changes in $Q$-factor of the BSTO BAW-SMR and the microstructure of the BSTO films deposited at different temperatures in the range $450-650{ }^{\circ} \mathrm{C}$. The analysis of the dielectric response of the BSTO films is used to calculate the defect density parameter and the effect of the amorphous layer at the bottom interface. The dependence of $Q$-factor on the BSTO film growth temperature reveals a sharp maximum at $590^{\circ} \mathrm{C}$ with $Q$-factors up to 400 at $5.2 \mathrm{GHz}$. The observed increase in the $Q$-factor with growth temperature is associated with the following changes in the BSTO film microstructure and corresponding extrinsic acoustic losses: i) decrease in density of oxygen vacancies and corresponding loss caused by Rayleigh scattering at localized defects; ii) decrease in thickness of the amorphous layer at the interface between the BSTO film and Pt bottom electrode and corresponding acoustic attenuation; and iii) reduction in the BSTO(111) texture misalignment and corresponding loss caused by the generation of shear waves leaking into the substrate. The observed drop in the $Q$-factor with a growth temperature above $590^{\circ} \mathrm{C}$ is associated with: i) sharp increase in the roughness at interfaces and corresponding acoustic losses due to waves scattering and resonance broadening by local thickness variations; ii) increase in the $\mathrm{BSTO}(111)$ texture misalignment and corresponding loss caused by the generation of shear waves; and iii) deterioration of reflection performance of the Bragg reflector due to recrystallization of the $\mathrm{SiO}_{2}$ layers, partial oxidation of the $\mathrm{W}$ layers, and a corresponding increase in the loss associated with waves leaking into the substrate. An acoustoelectric modeling shows that the generation of shear waves leaking through the Bragg reflector is likely not a dominant loss mechanism in our BSTO BAW-SMR. The trend of the steep decrease in the $Q$-factor with growth temperature above $590^{\circ} \mathrm{C}$ can be well described by a proposed model of loss associated with the diffuse scattering of the acoustic energy from the rough interface. Thus, we assume that the waves scattering by surface roughness at interfaces is the main loss mechanism currently limiting the $Q$-factor of our BSTO BAW-SMR. The increase in roughness at both top and bottom interfaces of the BSTO films grown above $590^{\circ} \mathrm{C}$ are caused by a deformation of the Pt bottom electrode, which most likely takes place during heating up due to large differences in the thermal expansion coefficients of the $\mathrm{Pt}$ and the $\mathrm{SiO}_{2}$ layer below. These findings can help to further improve the $Q$-factor by optimizing the BSTO films' growth conditions and the BAW-SMR design.

\section{ACKNOWLEDGMENTS}

This work was supported by the project VR FBAR of the Swedish Research Council.
${ }^{1}$ J. Berge, M. Norling, A. Vorobiev, and S. Gevorgian, J. Appl. Phys. 103, 064508 (2008).

${ }^{2}$ A. Noeth, T. Yamada, P. Muralt, A. K. Tagantsev, and N. Setter, IEEE Trans. Ultrason. Ferroelectr. Freq. Control 57, 379 (2010).

${ }^{3}$ G. N. Saddik, D. S. Boesch, S. Stemmer, and R. York, IEEE MTT-S Int. Microwave Symp. Dig. 1, 1263 (2008).

${ }^{4}$ X. Zhu, J. D. Phillips, and A. Mortazawi, IEEE MTT-S Int. Microwave Symp. Dig. 1, 671 (2007).

${ }^{5}$ K.-Y. Hashimoto, RF Bulk Acoustic Wave Filters for Communications (Artech House, Norwood, MA, 2009).

${ }^{6}$ R. Nava, R. Callarotti, H. Ceva, and A. Martinet, Phys. Rev. 188, 1456 (1969).

${ }^{7}$ J. F. Rosenbaum, Bulk Acoustic Wave Theory and Devices (Artech House, Norwood, MA, 1988).

${ }^{8}$ A. Volatier, E. Defay, M. Aid, A. N'hari, P. Ancey, and B. Dubus, Appl. Phys. Lett. 92, 032906 (2008).

${ }^{9}$ R. E. Green, Ultrasonic Investigation of Mechanical Properties (Academic, New York, 1973).

${ }^{10}$ J. Berge, A. Vorobiev, W. Steichen, and S. Gevorgian, IEEE Microw. Wirel. Compon. Lett. 17, 655 (2007).

${ }^{11}$ R. Thalhammer, J. Kaitila, R. Aigner, and S. Marksteiner, Proc.-IEEE Ultrason. Symp. 1, 282 (2004).

${ }^{12}$ J. Kaitila, IEEE Ultrason. Symp. Dig. 1, 120 (2007).

${ }^{13}$ S. Marksteiner, J. Kaitila, G. G. Fattinger, R. Aigner, Proc.-IEEE Ultrason. Symp. 1, 329 (2005).

${ }^{14}$ F. Martin, M.-E. Jan, B. Belgacem, M.-A. Dubois, and P. Muralt, Thin Solid Films 514, 341 (2006).

${ }^{15}$ J. Bjurström, G. Wingqvist, and I. Katardjiev, IEEE Ultrason. Symp. Dig. 1, 321 (2005).

${ }^{16}$ A. Vorobiev and S. Gevorgian, Appl. Phys. Lett. 96, 212904 (2010).

${ }^{17} \mathrm{M}$. Norling, J. Berge, and S. Gevorgian, IEEE MTT-S Int. Microwave Symp. Dig. 1, 101 (2009).

${ }^{18}$ A. Vorobiev and S. Gevorgian, paper presented at MEMSWAVE 2010, Otranto, Italy, 28-30 June 2010.

${ }^{19}$ A. K. Tagantsev, V. O. Sherman, K. F. Astafiev, J. Venkatesh, and N. Setter, J. Electroceramics 11, 5 (2003).

${ }^{20}$ K. Bethe, Philips Res. Rep. (Suppl 2) 2, 73 (1970).

${ }^{21}$ Z. Ma, A. J. Becker, P. Polakos, H. Huggins, J. Pastalan, H. Wu, Y. H. Wong, and P. Mankiewich, IEEE Trans. on Electron Devices 45, 1811 (1998).

${ }^{22}$ D.-H. Kim, M. Yim, D. Chai, J.-S. Park, and G. Yoon, Jpn. J. Appl. Phys. 43, 1545 (2004).

${ }^{23}$ K. Kuribayashi and S. Kitamura, Thin Solid Films 400, 160 (2001).

${ }^{24}$ P. G. Klemens, Proc. Phys. Soc., London, Sect. A 68, 1113 (1955).

${ }^{25}$ M. Aspelmeyer, U. Klemradt, W. Hartner, H. Bachhofer, and G. Schindler, J. Phys. D: Appl. Phys. 34, A173 (2001).

${ }^{26}$ O. G. Vendik, E. K. Hollmann, A. B. Kozyrev, and A. M. Prudan, J. Supercond. 12, 325 (1999).

${ }^{27}$ O. G. Vendik and S. P. Zubko, J. Appl. Phys. 88, 5343 (2000).

${ }^{28}$ O. G. Vendik and L. T. Ter-Martirosyan, J. Appl. Phys. 87, 1435 (2000).

${ }^{29}$ S. Saha and S. B. Krupanidhi, Appl. Phys. Lett. 79, 111 (2001).

${ }^{30}$ M. S. Tsai and T. Y. Tseng, Mater. Chem. Phys. 57, 47 (1998).

${ }^{31}$ N. M. Alford, P. K. Petrov, A. G. Gagarin, A. B. Kozyrev, A. I. Sokolov, O. I. Soldatenkov, and V. A. Volpyas, Appl. Phys. Lett. 87, 222904 (2005).

${ }^{32}$ Yu. A. Boikov, B. M. Goltsman, V. K. Yarmarkin, and V. V. Lemanov, Appl. Phys. Lett. 78, 3866 (2001).

${ }^{33}$ D. Muller-Sajak, A. Cosceev, C. Brand, K. R. Hofmann, and H. Pfnur, Phys. Status Solidi C 7, 316 (2010).

${ }^{34}$ W. G. Lee, S. I. Woo, J. C. Kim, S. H. Choi, and K. H. Oh, Thin Solid Films 237, 105 (1994).

${ }^{35}$ W. Schirmacher, G. Diezemann, and C. Ganter, Phys. Rev. Lett. 81, 136 (1998).

${ }^{36}$ T. S. Grigera, V. Martin-Mayor, G. Parisi, and P. Verrocchio, Phys. Rev. Lett. 87, 085502 (2001).

${ }^{37}$ G. F. Iriarte, F. Engelmark, and I. V. Katardjiev, J. Mater. Res. 17, 1469 (2002).

${ }^{38}$ S. N. Ivanov and Ye. N. Khazanov, Radio Eng. Electron. 26, 133 (1981).

${ }^{39}$ R. O. Bell and G. Rupprecht, Phys. Rev. 129, 90 (1963). 